ОСОБЕННОСТИ ПОВЕДЕНИЯ МИКРОВОЛНОВОГО ИЗЛУЧЕНИЯ АКТИВНЫХ ОБЛАСТЕЙ НА СОЛНЦЕ ПЕРЕД КОРОНАЛЬНЫМИ ВЫБРОСАМИ МАССЫ

\author{
Бакунина И.А. ${ }^{1}$, Мельников В.Ф. ${ }^{2}$, Абрамов-Максимов В.Е. ${ }^{2}$, \\ Моргачев A.C. ${ }^{3}$, Шаин A.B. ${ }^{2}$ \\ ${ }^{1}$ Национальный исследовательский университет «Высшая школа экономики», \\ Нижний Новгород, Россия \\ ${ }^{2}$ Главная (Пулковская) астрономическая обсерватория РАН, Санкт-Петербург, Россия \\ ${ }^{3}$ Нижегородский государственный университет им. Н.И. Лобачевского, \\ Нижний Новгород, Россия
}

\title{
PECULIARITIES OF THE MICROWAVE EMISSION BEHAVIOR IN SOLAR ACTIVE REGIONS BEFORE CORONAL MASS EJECTIONS
}

\author{
Bakunina I.A. ${ }^{1}$, Melnikov V.F. ${ }^{2}$, Abramov-Maximov V.E. ${ }^{2}$, \\ Morgachev A.S. ${ }^{3}$, Shain A.V. ${ }^{2}$ \\ ${ }^{1}$ National Research University Higher School of Economics, Nizhny Novgorod, Russia \\ ${ }^{2}$ Pulkovo Observatory, St. Petersburg, Russia \\ ${ }^{3}$ Lobachevsky State University, Nizhniy Novgorod, Russia
}

Solar flares and coronal mass ejections (CME) are the most powerful manifestations of solar activity. Both phenomena associated with the evolution of the spatial structure of the magnetic field of active regions (AR). It is known that not all powerful flares accompanied by CME. In some cases, CME are observed, associated with very low intensity bursts. At the same time, the observational signs that determine the ability of AR to cause the eruption of matter from AR into the high layers of the solar corona are still not clear. This makes it difficult to understand the physical mechanism of eruption initiation (CME trigger). The purpose of this work is to search for observational signs of the onset of the eruptive process. For this, we conducted a comparative analysis of pre-flare and flare conditions for 6 flare events, accompanied by $C M E$, and 5 events not accompanied by CME.

\section{DOI: 10.31725/0552-5829-2020-15-18}

Солнечные вспышки и корональные выбросы массы (КВМ) являются наиболее мощными проявлениями солнечной активности. Считается, что оба явления связаны с эволюцией пространственной структуры магнитного поля активных областей (AО), в которых магнитная энергия постепенно накапливается в непотенциальной форме, а затем быстро выделяется в короне в результате нарушения равновесия или развития неустойчивости. Иногда мощные вспышки сопровождаются КВМ, их называют эруптивными. В некоторых случаях наблюдаются КВМ, ассоциированные со вспышками очень низкой интенсивности. Но в большинстве случаев вспышки не сопровождаются КВМ и их называют неэруптивными («соnfined»). Важной задачей является найти теоретические и наблюдательные 
признаки, определяющие способность АО вызывать эрупцию вещества из АО в высокие слои солнечной короны.

В последние годы заметных успехов достигло теоретическое моделирование этих явлений. Накопление магнитной энергии связывают в основном с тремя процессами: всплытие магнитного потока, обладающего спиральностью; фотосферные сдвиги; вращение солнечных пятен ([1]). Процесс выделения свободной энергии приписывают неустойчивостям в токовых слоях, которые могут образоваться либо при взаимодействии соседних трубок магнитного поля, либо в результате вытягивания силовых линий в результате выброса магнитного жгута вверх в корону (стандартная модель вспышки). В последние годы популярными стали модели энерговыделения и выброса корональной массы благодаря тороидальной [2] и изгибной неустойчивостям ([3]). Недавно Ishiguro and Kusano [4] предложили так называемую неустойчивость сдвоенной арки (DAI), чтобы инициировать процесс пересоединения, требуемый для поднятия магнитного жгута и перевода его в неустойчивый режим. В работе [5] на основе анализа результатов экстраполяции магнитного поля в корону в приближении нелинейного бессилового поля (NLFFF), было показано, что вспышка произошла в результате взаимодействия двух систем скрученных магнитных петель с большим широм (около 80 градусов) над линией инверсии магнитной полярности. Отметим, что хотя и показано, что сильная скрученность магнитных жгутов - это свойство, связанное с возникновением вспышек, но оно не является достаточным условием для возникновения эрупций и КВМ. Требуется изучить дополнительные факторы, влияющие на сдерживание и развитие процессов эрупции.

Важность физических процессов, происходящих в области пересекающихся магнитных петель, подтверждается недавним сравнительным анализом пространственной динамики УФ и микроволнового излучения, проведенного для нескольких вспышек $[6,7]$. Было обнаружено явление сильной нестационарности микроволновых источников, связанное с появлением за 1-3 часа до вспышки пересекающихся друг с другом ярких и "горячих" (Т 10 млн К) ультрафиолетовых петель.

Целью настоящей работы является поиск в микроволновом диапазоне отличительных наблюдательных признаков между началом эруптивных и неэруптивных вспышек.

\section{Данные наблюдений}

Для проведения предварительного сравнительного анализа условий формирования КВМ мы отобрали 6 вспышечных событий, сопровождавшихся КВМ, и 5 событий, не сопровождавшихся КВМ (таблица 1). Все события относятся к периоду 2011-2014 гг. и наблюдались Радиогелиографом Нобеяма. Часть активных областей из этих выборок наблюдалась также на спектрополяриметре радиотелескопа РАТАН-600. 
Таблица 1.

\begin{tabular}{|l|l|l|l|lrc|}
\hline $\begin{array}{l}\text { Flare Peak Time } \\
\text { Flare Class }\end{array}$ & NOAA & AR Position & E/C & \multicolumn{2}{|l|}{ FoRH } & \\
& & & & F17(SFU) & Tb(K) & Dur(sec) \\
\hline 2011-07-30 T02:09 M9.3 & 11261 & S20W10 & C & 569 & $5.8 \mathrm{e}+07$ & 594 \\
\hline 2011-09-06 T01:50 M5.3 & 11283 & N14W07 & E & 206 & $2.0 \mathrm{e}+07$ & 3770 \\
\hline 2011-10-02 T00:50 M3.9 & 11305 & N12W26 & C & 42 & $2.2 \mathrm{e}+06$ & 4149 \\
\hline 2012-01-23 T03:59 M8.7 & 11402 & N28W21 & E & 3703 & $7.4 \mathrm{e}+07$ & 16207 \\
\hline 2012-03-09 T03:53 M6.3 & 11429 & N15W03 & E & 90 & $3.9 \mathrm{e}+06$ & 4986 \\
\hline 2012-05-10 T04:18 M5.7 & 11476 & N12E22 & C & 54 & $5.2 \mathrm{e}+06$ & 363 \\
\hline 2013-10-24 T00:30 M9.3 & 11877 & S09E10 & E & 78 & $2.9 \mathrm{e}+06$ & 1042 \\
\hline 2013-11-03 T05:22 M4.9 & 11884 & S12W17 & C & 233 & $1.3 \mathrm{e}+07$ & 4203 \\
\hline 2014-02-04 T04:00 M5.2 & 11967 & S14W06 & C & 3572 & $3 \mathrm{e}+07$ & 1556 \\
\hline $2014-09-28$ T02:58 M5.1 & 12173 & S13W23 & E & 373 & $2 \mathrm{e}+06$ & 3118 \\
\hline $2014-12-17$ T04:51 M8.7 & 12242 & S18E08 & E & 236 & $8.0 \mathrm{e}+06$ & 8808 \\
\hline
\end{tabular}

$B$ таблице 1: крайняя левая колонка - данные о дате и времени вспышки и ее рентгеновском балле; в четвёртой колонке Е обозначает эруптивную вспышку (eruptive), $\mathrm{C}$ - неэруптивную (confined); в крайней правой приведены параметры радиоизлучения по данным Радиогелиографа Нобеяма: поток, яркостная температура и длительность вспышки.

\section{Результаты анализа}

Из таблицы 1 видно, что в обеих выборках событий “Е” (эруптивные) и "С" (неэруптивные) присутствуют только вспышки класса М. Сравнение выбранных трех параметров радиоизлучения для выборок “Е” или “C” не выявило заметных отличий в потоке, яркостной температуре и длительности вспышки. Анализ динамики радиоизлучения перед вспышками показал, что сильная нестационарность радиоизлучения перед вспышками имеет место в обеих выборках. Аналогично результатам работ [6-8], получено, что чем мощнее вспышка, тем более вероятны субвсплески и усиление цугов колебаний радиоизлучения перед ней [8].

Предварительный анализ микроволновых спектров, наблюдаемых на РАТАН-600, показал, что динамика частотного спектра микроволнового излучения, может заметно отличаться перед разными типами вспышек, эруптивными и неэруптивными. В качестве иллюстрации на рис. 1 показаны частотные спектры, наблюдаемые перед двумя такими вспышками. Эруптивное событие NOAA 11158: рост потока произошёл во всём частотном диапазоне, что свидетельствует, скорее всего, об увеличении площади излучающего радиоисточника. Неэруптивное событие NOAA 11261: рост потока произошёл только на низких частотах. На высоких частотах, где за интенсивность излучения отвечают области с более сильным магнитным полем, поток практически не вырос, что свидетельствует, скорее всего, о том, что новые области с сильным полем не появились. В то же время площадь радиоисточников со слабым полем явно увеличилась. 

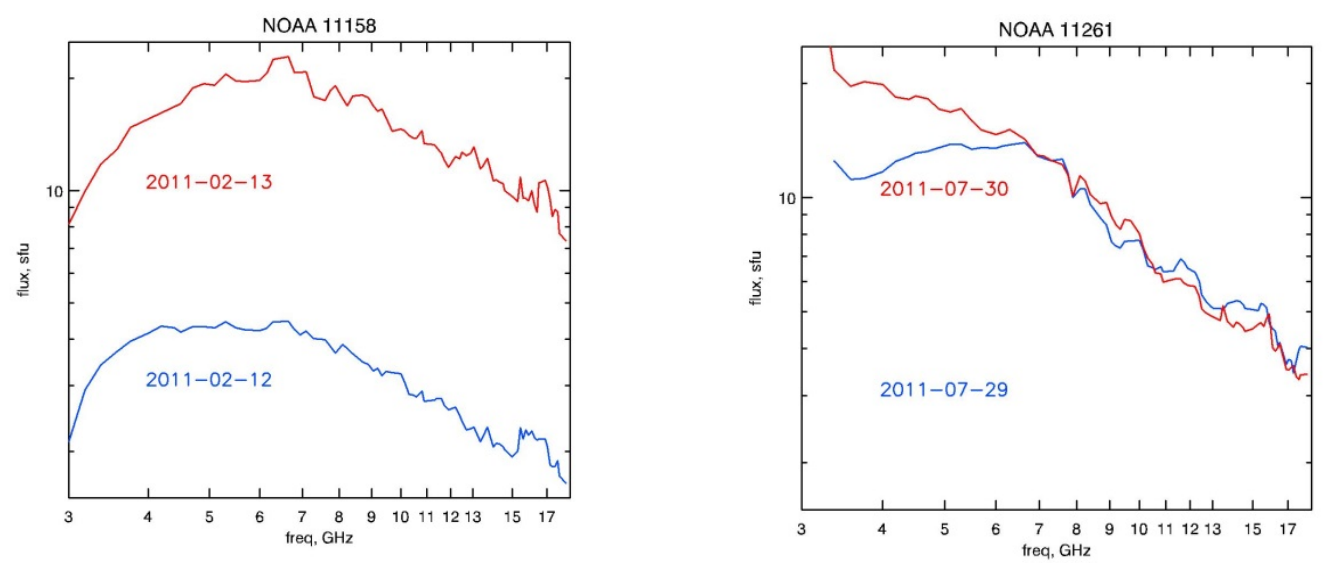

Рис. 1. Различие в динамике частотного спектра микроволнового излучения перед разными типами вспышек - эруптивной (слева) и неэруптивной (справа).

Данная работа является началом сравнительного исследования поведения характеристик микроволнового излучения эруптивных и неэруптивных вспышек. Следующим шагом будет проведение детального анализа пространственной структуры и динамики радиоисточников за несколько часов до вспышки с привлечением данных в ультрафиолетовом диапазоне и магнитограмм SDO/HMI, а также восстановленных данных о 3D-структуре коронального магнитного поля и соответствующих электрических токов.

Работа выполнена при поддержке грантов РФФИ №№ 17-52-80064, $18-02-00856$.

\section{Литература}

1. Lin P.H., Kusano K., Shiota D. // ApJ, 2020, 894, 20

2. Kliem, B., \&Török, T. // PhRvL, 2006, 96, 255002

3. Liu, R., Kliem, B., Titov, V.S., et al. // ApJ, 2016, 818, 148

4. Ishiguro, N. \& Kusano, K. // ApJ, 2017, 843, 101

5. Sharykin I.N., Zimovets I.V., Myshyakov I.I. // ApJ, 2020, 893:159 (25 pp.)

6. Bakunina, I.A., Melnikov V.F. // AApTr, 2019, 31, Issue 3, 251

7. Bakunina, I.A., Melnikov, V.F., Morgachev, A.S. // Astrophysics, 2020, 63, No. 2, 252

8. Абрамов-Максимов В.Е., Бакунина И.А. // Ядерная физика, 2018, 81, N 3, с. 366. 